\title{
Melanocitosis dérmica adquirida en la mano de un paciente pediátrico
}

\author{
Eduardo Marín-Hernández ${ }^{1 *}$, Eduardo J. Marín-González ${ }^{2}$ y Georgina Siordia-Reyes ${ }^{3}$

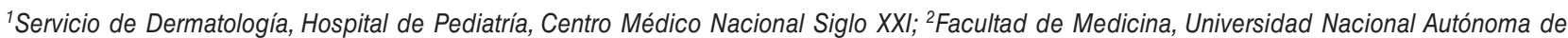 \\ México; ${ }^{3}$ Servicio de Patología, Hospital de Pediatría, Centro Médico Nacional Siglo XXI. Ciudad de México, México
}

\begin{abstract}
Resumen
Introducción: Las melanocitosis dérmicas son un grupo de enfermedades dermatológicas pigmentarias asociadas con la proliferación melanocítica. Se clasifican con base en su número y localización profunda a nivel de la dermis; pueden ser congénitas o adquiridas. Caso clínico: Paciente de sexo masculino de 11 años de edad, sin antecedentes de importancia para el padecimiento actual. Inicio con mácula oscura en la palma de la mano izquierda hace 5 años, asintomática, de crecimiento paulatino. A la exploración física, se detectó dermatosis que afectaba la palma izquierda, cara palmar de las falanges proximales del tercer y cuarto dedos, caracterizada por la presencia de mácula grisácea negruzca, bordes difusos e irregulares, no infiltrada ni indurada. En la dermatoscopia se detectó un patrón de pigmento de tono gris acero con áreas de color café, y con evidencia de puntos blanquecinos dentro de estas. En la histopatología se identificaron células fusiformes, con núcleo grande y la presencia de pigmento melánico en su interior, con distribución perivascular y entremezclados con las fibras de colágeno en la dermis superficial y media. Con base en las características clínicas e histopatológicas de la lesión, se concluyó melanocitosis dérmica adquirida de la mano como el diagnóstico definitivo. Conclusiones: Los reportes de casos de melanocitosis dérmica adquiridas atípicas son infrecuentes. La melanocitosis dérmica de la mano es una variante de estas enfermedades, de la que existen menos de 10 casos. Se presenta el primer caso reportado en Latinoamérica hasta el momento, con el objetivo de ampliar el conocimiento de sus características clínico-histológicas y dermatoscópicas.
\end{abstract}

Palabras clave: Melanocitosis dérmica. Mano. Escolar.

\section{Acquired dermal melanocytosis on the hand of a pediatric patient}

\begin{abstract}
Background: Dermal melanocytosis is a group of pigmentary dermatological diseases associated with melanocytic proliferation, which are classified based on their number and depth at the level of the dermis; they may be congenital or acquired. Case report: An 11-year-old male patient with no history of importance for the current condition started 5 years ago with a dark macula in the left hand palm, which was asymptomatic but grew gradually. On physical examination, dermatoses affecting
\end{abstract}

*Eduardo Marín-Hernández

E-mail: emarinh1973@yahoo.com.mx

DOI: 10.24875/BMHIM.19000135
Fecha de aceptación: 23-09-2019
Fecha de recepción: 26-08-2019
Disponible en internet: 13-11-2019 Bol Med Hosp Infant Mex. 2019;76:294-297 www.bmhim.com 
the left palm, palmar face of proximal phalanges of the third, fourth and fifth fingers, characterized by the presence of blackish greyish macula, diffuse and irregular edges, not infiltrated or indurated were detected. The dermatoscopy identified a pattern of pigment with a greyish-brown tone with brown areas, showing whitish spots inside. In the histopathology, the presence of spindle cells was observed in the superficial and middle dermis, with a large nucleus and the presence of a melanic pigment inside, with perivascular distribution and intermingled with the collagen fibres. Based on the clinical characteristics and the histopathological findings, acquired dermal melanocytosis of the hand was concluded as the final diagnosis. Conclusions: Case reports of atypical acquired dermal melanocytosis are infrequent. Dermal melanocytosis of the hand is a rare variant of these diseases, of which less than 10 cases have been reported. At present, this case of dermal melanocytosis is the first reported in Latin America with the aim to extend the knowledge of its clinical-histological and dermatoscopic characteristics.

Key words: Dermal melanocytosis. Acquired. Hand. Grade-schooler.

\section{Introducción}

Las melanocitosis dérmicas (MD) corresponden a un término histológico que también es usado para describir un espectro clínico de enfermedades cutáneas ${ }^{1}$ asociadas con la proliferación melanocítica, con base en su número y su localización profunda a nivel de la dermis $^{2}$. Se han descrito numerosas variantes morfológicas de la melanocitosis dérmica, de las cuales el nevo azul, la mancha mongólica, el nevo de Ota y el nevo de Ito son las más comunes ${ }^{3}$. Sin embargo, se han publicado otras formas atípicas de melanocitosis dérmica, ya que, por su localización anatómica, no se adaptan en su descripción a las formas clásicas mencionadas.

Se reporta el caso de un paciente de sexo masculino en etapa escolar que presentó melanocitosis dérmica adquirida localizada en la mano, lo que representa una circunstancia clínica infrecuente.

\section{Caso clínico}

Paciente de sexo masculino de 11 años de edad, sin antecedentes de importancia para el padecimiento actual. Fototipo III en la escala de Fitzpatrick. Negó traumatismos. A referencia de la madre, inició con mácula oscura en la palma de la mano izquierda a la edad de 6 años, asintomática, de crecimiento paulatino. Fue evaluado por un dermatólogo, quien diagnosticó hemangioma a descartar nevo, motivo de envío al Servicio de Patología.

Ante la exploración física, se observó dermatosis que afecta la palma izquierda, cara palmar de las falanges proximales del tercer y cuarto dedos, caracterizada por la presencia de una mácula grisácea negruzca de $4 \mathrm{~cm}$, con bordes difusos e irregulares, no infiltrada ni indurada (Fig. 1). En la dermatoscopia se identificó un patrón de pigmento de tono gris acero con áreas en color café, y se evidenciaban puntos blanquecinos

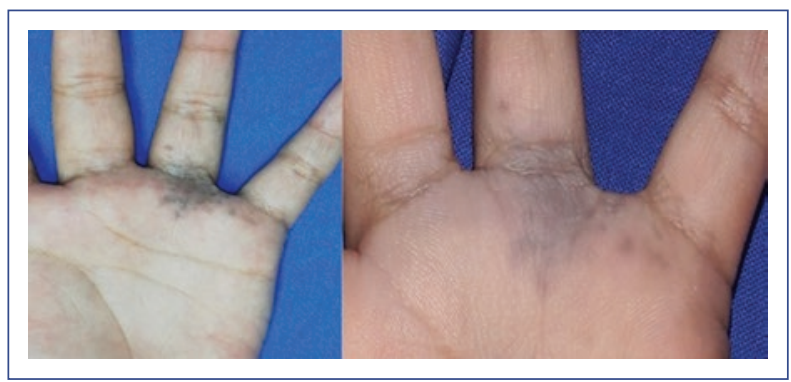

Figura 1. Dermatosis con tono pizarra grisáceo y bordes difusos.

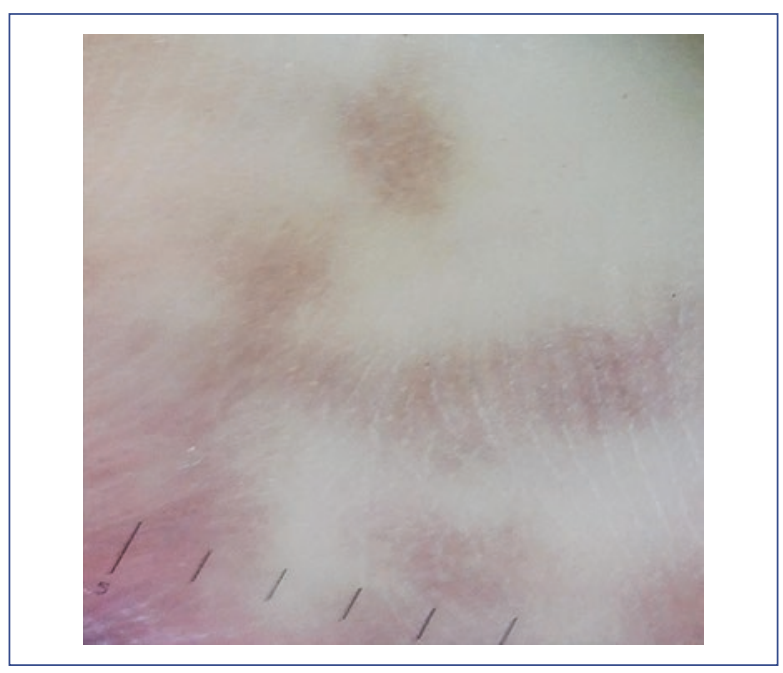

Figura 2. Patrón de pigmento de tono gris acero con áreas en color café de la dermatosis.

dentro de estas (Fig. 2). Con estos hallazgos morfológicos, se realizó biopsia, con diagnóstico clínico de nevo, a descartar proceso neoplásico.

En la histopatología se identificó epidermis con hiperqueratosis y acantosis discreta (Fig. $3 \mathrm{~A}$ ). A nivel de 
Tabla 1. Melanocitosis dérmicas clásicas

\begin{tabular}{|l|l|l|l|l|}
\hline & Nevo de Ota & Nevo de lto & Manchas mongólicas & $\begin{array}{l}\text { Hamartoma melanocítico } \\
\text { dérmico }\end{array}$ \\
\hline $\begin{array}{l}\text { Predominio de } \\
\text { sexo }\end{array}$ & Mujeres & Mujeres & Sin predominio & Sin predominio \\
\hline Distribución & Trigeminal & Acromioclavicular & $\begin{array}{l}\text { Sacro (más } \\
\text { comúnmente) }\end{array}$ & Dermatoma \\
\hline $\begin{array}{l}\text { Potencial de } \\
\text { malignidad }\end{array}$ & $\begin{array}{l}\text { Raro: SNC, ocular y } \\
\text { cutáneo }\end{array}$ & Raro: cutáneo & No reportado & No reportado \\
\hline Histología & $\begin{array}{l}\text { Proliferación de } \\
\text { melanocitos en forma de } \\
\text { bandas }\end{array}$ & $\begin{array}{l}\text { Proliferación de } \\
\text { melanocitos en forma de } \\
\text { bandas }\end{array}$ & $\begin{array}{l}\text { Sin reacción fibrótica en } \\
\text { el estroma }\end{array}$ & $\begin{array}{l}\text { Moderada presencia de } \\
\text { melanocitos dendríticos, } \\
\text { principalmente en la dermis } \\
\text { reticular superficial }\end{array}$ \\
\hline Pronóstico & $\begin{array}{l}\text { No presenta involución } \\
\text { espontanea; puede } \\
\text { agrandarse después de } \\
\text { la pubertad }\end{array}$ & $\begin{array}{l}\text { No presenta involución } \\
\text { espontanea; puede } \\
\text { agrandarse después de } \\
\text { la pubertad }\end{array}$ & $\begin{array}{l}\text { Se aclara con la edad. } \\
\text { Cuando se asocia con } \\
\text { algún error innato del } \\
\text { metabolismo, puede } \\
\text { progresar }\end{array}$ & \begin{tabular}{l} 
La resolución es variable \\
\hline
\end{tabular}
\end{tabular}

SNC: sistema nervioso central.

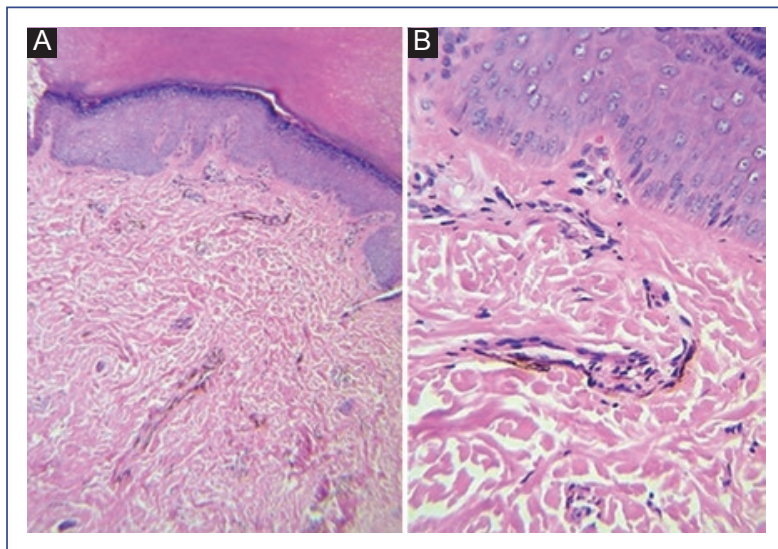

Figura 3. Histopatología. A: Epidermis con hiperqueratosis y acantosis discreta. B: Células alargadas, pigmentadas, entre los haces de colágeno y con una distribución perivascular.

la dermis superficial y media, se detectaron células fusiformes, con núcleo grande y pigmento melánico en su interior, con distribución perivascular y entremezclados con las fibras de colágeno (Fig. 3 B). Con base en las características clínicas y los hallazgos histopatológicos, se concluyó melanocitosis dérmica adquirida en la mano como diagnóstico definitivo.

\section{Discusión}

Los melanocitos son originados embriológicamente a partir de las células de la cresta neural (CCN), que derivan de la capa ectodérmica. Estudios en ratones indican que las CCN se inician como células pluripotenciales, capaces de diferenciarse en múltiples tipos de células, incluyendo neuronas, osteocitos, células musculares, condrocitos, células glía y melanocitos. Los melanocitos pueblan la epidermis folicular e interfolicular por dos vías migratorias: la vía dorsolateral y la vía dorsoventral. Se cree que las $C C N$ que migran en la vía dorsolateral mediante la llamada primera ola, originan la mayor parte de los melanocitos cutáneos. Estas células se diferencian en melanoblastos, los cuales proliferan en la región conocida como el área de migración, localizada entre el ectodermo, el tubo neural y las somitas. Los melanoblastos continúan proliferando, diferenciándose hacia la dermis y la epidermis; al llegar a la epidermis, la diferenciación se completa. Una fuente alternativa de melanocitos cutáneos referida como la segunda ola de migración melanocítica, se acrecienta en los precursores de células de Schwann asociados con los nervios periféricos y ocurre en la vía dorso ventral. En humanos, esta migración comienza a las dos semanas y media de gestación, y termina alrededor de la semana ocho ${ }^{2,4}$. El mecanismo de la migración melanocítica aún es pobremente entendido, pero se piensa que involucra moléculas como la fascina y $R a c 1^{5}$.

El concepto de MD abarca una serie de entidades dermatológicas pigmentarias asociadas con la proliferación melanocítica, que se clasifican con base en su número y localización profunda a nivel de la dermis ${ }^{6}$. 
Se tiene la hipótesis de que las MD son el resultado de la detención de los melanocitos en la dermis, lo que permite observar un aumento en el número de estas células en pacientes con este padecimiento?

Las MD se dividen en dos grupos principales: las clásicas y las atípicas. En el primer grupo se considera el nevo de Ota, el nevo de Ito, la mancha mongólica y el hamartoma melanocítico dérmico. En general, este tipo de enfermedades se presenta con mayor frecuencia en población de ascendencia asiática y típicamente en niños, sin predominio de $\operatorname{sex}^{8}$. Sus principales características se mencionan en la tabla 1.

Junto con estos cuadros de MD, se han descrito algunos casos aislados que han contribuido a ampliar la lista ya referida. Entre ellos, cabe destacar el nevo de Hidano, nevo de Hori, naevus fuscocaeruleus zygomaticus o nevo de Sun y el anillo melanótico periorbital de Watanabe ${ }^{4,9}$. En 1977 se describieron cuatro pacientes con melanocitosis dérmica de cara y extremidades $^{10}$. Fukuda, et al., en 1993, realizaron el primer reporte utilizando el término de melanocitosis dérmica adquirida de la mano ${ }^{11}$. A partir de este y al día de hoy, se han reportado alrededor de ocho casos (incluyendo el presente caso) en la literatura mundial con esta variante clínica de $\mathrm{MD}^{12}$. De estos casos, cuatro son de inicio en la edad pediátrica. Esta entidad no presenta predilección por el sexo. De los casos reportados, la mayoría son en población asiática y solamente uno fue en un paciente hispano con fototipo III en la clasificación de Fitzpatrick, y afectaban la cara dorsal, palmar 0 ambas de la mano.

Aun cuando la fisiopatología resulta desconocida, se propone que se debe a la reactivación de melanocitos dérmicos latentes por factores como el trauma local, inflamación o exposición a químicos ${ }^{13}$. En general, debido a su benignidad, las melanocitosis dérmicas adquiridas usualmente no requieren tratamiento ${ }^{6-8}$. Las indicaciones generales se basan en evitar la exposición solar. Las lesiones que estéticamente el paciente considere inaceptables pueden ser manejadas mediante laser de tipo Nd: YAG pulsado (por sus siglas en inglés, neodymium-doped yttrium aluminium garnet) ${ }^{7}$.

La trascendencia de este reporte radica en sumar un nuevo caso de melanocitosis dérmica adquirida de la mano a la literatura, que hasta el momento corresponde al paciente más joven con MD y el primer caso de este padecimiento reportado en Latinoamérica, con el objetivo de ampliar el conocimiento de las características clínico-histológicas y dermatoscópicas de las MDM.

\section{Responsabilidades éticas}

Protección de personas y animales. Los autores declaran que para esta investigación no se han realizado experimentos en seres humanos ni en animales.

Confidencialidad de los datos. Los autores declaran que han seguido los protocolos de su centro de trabajo sobre la publicación de datos de pacientes.

Derecho a la privacidad y consentimiento informado. Los autores han obtenido el consentimiento informado de los pacientes y/o sujetos referidos en el artículo. Este documento obra en poder del autor de correspondencia.

\section{Conflicto de intereses}

Los autores declaran no tener ningún conflicto de intereses.

\section{Financiamiento}

Ninguno.

\section{Agradecimientos}

Se agradece el interés y esfuerzo hacia la dermatología pediátrica, así como a este reporte.

\section{Bibliografía}

1. Mosher DB, Fitzpatrick TB, Hori Y, Ortonne JP. Disorders of Pigmentation. En: Fitzpatrick TB, Eisen AZ, Wolff K, Freeberg IM, Austen KF, editores. Dermatology in general medicine. New York NY: McGraw-Hill; 199. pp:903-5.

2. Franceschini D, Dinulos J. Dermal melanocitosis and associated disorders. Curr Opin Pediatr. 2015;27:480-5.

3. Pastor MA, Vargas-Machuca I, Carrasco L, Izquierdo MJ, Fariña MC, Martín L, et al. Melanocitosis dérmica de la parte alta de la espalda. Actas Dermosifiliogr. 2002;93:404-5.

4. Rook A, Wilkinson DS, Ebling FJG, editores. Disorders of Skin Colour. En: Textbook of dermatology. Oxford: Blackwell Scientific Publications; 1998. p. $1753-815$.

5. Li A. The biology of melanocyte and melanocyte stem cell. Acta Biochim Biophys Sin. 2014;46:255-60.

6. Aguilar L. Nevus melanocítico en la infancia. An Esp Pediatr. 2001;54:477-83.

7. Bolognia JL, Jorizzo JL, Schaffer JV. Dermatología. Principales diagnósticos y tratamientos. Madrid: Editorial Mosby; 2004. pp. 1709-85.

8. Valdés F, Ginarte M, Toribio J. Melanocitosis dérmicas. Actas Dermosifiliograf. 2001;92:379-88

9. Cabrera HN, Hermida D, Griffa E, Carriquiri MF, García S. Nevo de Hori. Presentación de un caso y revisión de las melanocitosis dérmicas faciales névicas. Dermatol Arg. 2016;22:203-6.

10. Mevorah B, Frenk E, Delacrétaz J. Dermal melanocytosis. Report of an unusual case. Dermatologica. 1977:154:107-14.

11. Fukuda M, Kitajima J, Fushida H, Hamada T. Acquired dermal melanocytosis of the hand: a new clinical type of dermal melanocytosis. J Dermatol. 1993;20:561-5.

12. Young Jun J, Jeong Byun $\mathrm{H}$, Jin Oh $\mathrm{S}$, Hwan Oh S, Hye Park J, Hee Lee J. Acquired dermal melanocytosis confined to the hand. Ann Dermatol. 2018;30:380-1.

13. Permatasari F, Zhou BR, Luo D. Late-oset acquired dermal melanocitosis on the hand of a Chinese woman. Indian J Dermatol Venereol Leprol. 2013;79:269. 